\title{
ON THE ROW CONVERGENCE OF THE WALSH ARRAY FOR MEROMORPHIC FUNCTIONS
}

\author{
BY
}

E. B. $\operatorname{SAFF}\left({ }^{1}\right)$

1. Introduction. Let the function $f(z)$ be continuous on a compact set $E$ of the $z$-plane. If $E$ has no isolated points there exists [1, $\$ 12.3]$ for each pair of nonnegative integers $(n, \mu)$ a rational function $W_{n \mu}(z)$ of type $(n, \mu)$, i.e., a function of the form

$$
\frac{a_{n} z^{n}+a_{n-1} z^{n-1}+\cdots+a_{0}}{b_{\mu} z^{\mu}+b_{\mu-1} z^{\mu-1}+\cdots+b_{0}}, \quad \sum_{0}^{\mu}\left|b_{k}\right| \neq 0
$$

which is of best uniform approximation to $f(z)$ on $E$ in the sense that for all rational functions $r_{n \mu}(z)$ of type $(n, \mu)$ we have

$$
E_{n u}(f) \equiv\left[\max \left|f(z)-W_{n \mu}(z)\right| ; z \text { on } E\right] \leqq\left[\max \left|f(z)-r_{n \mu}(z)\right| ; z \text { on } E\right] \text {. }
$$

The $W_{n u}(z)$ need not be unique but any particular determination of them will suffice for our purposes.

The $W_{n \mu}(z)$ form a table of double entry

$$
\begin{array}{cccc}
W_{00}(z) & W_{10}(z) & W_{20}(z) & \ldots \\
W_{01}(z) & W_{11}(z) & W_{21}(z) & \ldots \\
W_{02}(z) & W_{12}(z) & \ldots & \ldots
\end{array}
$$

known as the Walsh array [2] which is similar in form and properties to the table of Padé. Indeed, J. L. Walsh has for the rows of his array established [3, p. 3] the following analogue of the important result [4] of Montessus de Ballore concerning the convergence of the rows of the Padé table:

THEOREM 1. Let $E$ be a compact set whose complement $K$ (with respect to the extended plane) is connected and possesses a classical Green's function $G(z)$ with pole at infinity. Let $\Gamma_{\sigma}(\sigma>1)$ denote generically the locus $G(z)=\log \sigma$ and let $E_{\sigma}$

Received by the editors May 28, 1969.

(1) The research of the author was supported by a Fulbright Grant at the Imperial College of Science and Technology, London, England.

Copyright (c) 1969, American Mathematical Society 
be the interior of $\Gamma_{\sigma}$. Suppose that the function $f(z)$ is analytic on $E$ and meromorphic with precisely $\mu$ poles (i.e., poles of total multiplicity $\mu$ ) in $E_{o}, 1<\rho \leqq \infty$. If $r_{n \mu}(z)$ is a sequence of rational functions of respective types $(n, \mu)$ which satisfy

$$
\underset{n \rightarrow \infty}{\lim \sup }\left[\max \left|f(z)-r_{n u}(z)\right| ; z \text { on } E\right]^{1 / n} \leqq 1 / \rho
$$

(a condition which is in particular satisfied by the $(\mu+1)$ th row of the Walsh array or $f(z)$ on $E$ ) the $n$ for $n$ sufficiently large each $r_{n u}(z)$ has precisely $\mu$ finite poles, which approach respectively the $\mu$ poles of $f(z)$ in $E_{\rho}$, and the $r_{n \mu}(z)$ converge uniformly to $f(z)$ on each compact subset of $E_{\rho}$ which contains no pole of $f(z)$.

Furthermore, if $f(z)$ has a singularity on $\Gamma_{o}$ then the equality sign holds in (1).

We note that if $f(z)$ is meromorphic with infinitely many poles in the finite plane and if no more than one pole of $f(z)$ lies on each $\Gamma_{\sigma}$, then Theorem 1 can be applied in turn to each row of the Walsh array. However if $f(z)$ has, say, $r(>1)$ poles on $\Gamma_{\rho}$, then the convergence properties of the rows $\mu+2$ through $\mu+r$ are not included in Theorem 1.

It is the aim of the present paper to give suitable hypotheses under which these rows do in fact converge. We shall prove in $\$ 3$ that if $E$ has a smooth boundary and the function $f(z)$ of Theorem 1 is analytic on $\Gamma_{\rho}$ except for a pole of order $r$ (>1) in the point $\alpha \in \Gamma_{o}(\alpha$ not a critical point of $G(z))$, then for constant $\nu, 0<\nu<r$, and $n$ sufficiently large each of the best approximating rational functions $W_{n, \mu+v}(z)$ has precisely $\mu+\nu$ finite poles, $\mu$ of which approach the $\mu$ poles of $f(z)$ in $E_{\rho}$ and $\nu$ of which approach the point $\alpha$. Consequently the $W_{n, \mu+v}(z)$ must converge uniformly to $f(z)$ on each compact subset of $E_{\rho}$ which contains no pole of $f(z)$. We show also, by methods recently used by the author [5], that the sequence $W_{n, u+v}(z)$ can converge in at most a finite number of points exterior to $\Gamma_{\rho}$. Furthermore we prove that

$$
0<A_{1} \leqq n^{1+2 v-r} \rho^{n} E_{n, \mu+v}(f) \leqq A_{2}<\infty, \quad n>0,
$$

and finally, in $\$ 4$, we show how the techniques of $\$ 3$ may be applied in certain cases where $f(z)$ has poles in more than one point on $\Gamma_{\rho}$.

As a basis for establishing the above results we define, in $\$ 2$, a special sequence of interpolating rational functions which have properties analogous to those established by R. Wilson [6], [7], [8] for the rows of the Padé table.

2. An interpolating sequence. Throughout the remainder of the paper we shall assume that the point set $E$ is the closed interior of a finite number of mutually exterior Jordan curves $C_{1}, C_{2}, \ldots, C_{m}$, where each $C_{j}$ is of class $A$, i.e., each $C_{j}$ can be represented parametrically in terms of arc length $s$ by $x=x(s), y=y(s)$, where $x(s)$ and $y(s)$ possess second derivatives with respect to $s$ which satisfy a Lipschitz condition of some positive order in $s$. We shall let $K, G(z), \Gamma_{\sigma}$, and $E_{\sigma}$ be as defined in Theorem 1, and further set $H(z) \equiv-\partial G(z) / \partial x+i \partial G(z) / \partial y$. 
Walsh has shown [9, Theorem 1] that for such a point set $E$ there exists a sequence of polynomials $\omega_{n}(z)$ which have respectively $n+1$ roots, all belonging to $\bigcup_{1}^{m} C_{j}$, and which satisfy

$$
\begin{gathered}
\left|\omega_{n}(z)\right| \leqq M e^{n g}, \quad z \text { on } E \\
\left|G(z)+g-n^{-1} \log \right| \omega_{n}(z)|| \leqq M n^{-1}
\end{gathered}
$$

for $z$ on each compact set exterior to $E$, where $e^{a}$ is the transfinite diameter of $E$.

Given a meromorphic function $F(z)$ which has a pole of order $r$ in the point $\alpha$ we define special rational functions $R_{n v}(z)$ of respective types $(n, v), \nu<r$, which interpolate to $F(z)$ in the $n+1$ roots of $\omega_{n}(z)$ and which satisfy $v$ additional constraints. Namely, the denominators of the $R_{n v}(z)$ shall be of the form

$$
Q_{n v}(z) \equiv(z-\alpha)^{v}+a_{n, v-1}(z-\alpha)^{v-1}+a_{n, v-2}(z-\alpha)^{v-2}+\cdots+a_{n 0},
$$

where the coefficients $a_{n k}$ are specified in

LEMMA 1. Let the integers $r, v$ satisfy $r>v \geqq 1$ and suppose $\alpha \in K$ is not a critical point of $G(z)$, i.e., $H(\alpha) \neq 0$. Let the polynomials $\omega_{n}(z)$ satisfy (2) and set

$$
\begin{aligned}
c_{n k} & \equiv \omega_{n}(\alpha) \theta_{n}^{(k)}(\alpha) / k !, & & \text { for } k \geqq 0, \\
& \equiv 0, & & \text { for } k<0,
\end{aligned}
$$

where $\theta_{n}(z) \equiv 1 / \omega_{n}(z)$. Then for $n$ sufficiently large the linear system of $v$ equations

$$
\sum_{k=0}^{\nu-1} x_{n k} c_{n, r-k-j}=-c_{n, r-\nu-j}, \quad 1 \leqq j \leqq \nu,
$$

in the $v$ unknowns $x_{n 0}, x_{n 1}, \ldots, x_{n, v-1}$ has a unique solution, say $x_{n k}=a_{n k}$, and for $k=0,1, \ldots, v-1$ we have

$$
\lim _{n \rightarrow \infty} n^{\nu-k} a_{n k}=(r-k-1) !(-1)^{\nu-k}\left(\begin{array}{l}
\nu \\
k
\end{array}\right) /(r-\nu-1) ! H(\alpha)^{\nu-k}
$$

Proof. We use the fact (see [5, proof of Theorem 1]) that

$$
\lim _{n \rightarrow \infty} n^{-k} \omega_{n}(\alpha) \theta_{n}^{(k)}(\alpha)=H(\alpha)^{k}, \quad k=0,1,2, \ldots
$$

Set $b_{k} \equiv 1 / k$ ! for $k \geqq 0$ and $b_{k} \equiv 0$ for $k<0$. For $t \geqq s \geqq 0$ let $A_{n}(t, s)$ denote the matrix

$$
A_{n}(t, s) \equiv\left[\begin{array}{cccc}
c_{n, t} & c_{n, t-1} & \cdots & c_{n, t-s} \\
c_{n, t-1} & c_{n, t-2} & \cdots & c_{n, t-s-1} \\
\vdots & \vdots & & \vdots \\
c_{n, t-s} & c_{n, t-s-1} & \cdots & c_{n, t-2 s}
\end{array}\right]
$$


and let $\Delta_{n}(t, s)$ be its determinant. Note that

$$
n^{(s-t)(s+1)} \Delta_{n}(t, s)=\left|\begin{array}{cccc}
n^{-t} c_{n, t} & n^{1-t} c_{n, t-1} & \cdots & n^{s-t} c_{n, t-s} \\
n^{1-t} c_{n, t-1} & n^{2-t} c_{n, t-2} & \cdots & n^{1+s-t} c_{n, t-s-1} \\
\vdots & \vdots & & \vdots \\
n^{s-t} c_{n, t-s} & n^{1+s-t} c_{n, t-s-1} & \cdots & n^{2 s-t} c_{n, t-2 s}
\end{array}\right|,
$$

and hence from (6) we obtain

$$
\lim _{n \rightarrow \infty} n^{(s-t)(s+1)} \Delta_{n}(t, s)=H(\alpha)^{(t-s)(s+1)} B(t, s),
$$

where

$$
B(t, s) \equiv\left|\begin{array}{cccc}
b_{t} & b_{t-1} & \cdots & b_{t-s} \\
b_{t-1} & b_{t-2} & \cdots & b_{t-s-1} \\
\vdots & \vdots & & \vdots \\
b_{t-s} & b_{t-s-1} & \cdots & b_{t-2 s}
\end{array}\right|
$$

Since $B(t, s) \neq 0$, i.e., the Padé table for $e^{z}$ is normal [10, p. 429], (7) implies that for $n$ sufficiently large $\Delta_{n}(t, s) \neq 0$, and so the first part of the lemma follows by taking $t=r-1, s=\nu-1$.

To prove (5) we first note that $\lim _{n \rightarrow \infty} n^{\nu-k} a_{n k}$ exists. Indeed, for $k=0$ we have

$$
a_{n 0}=(-1)^{v} \Delta_{n}(r-2, v-1) / \Delta_{n}(r-1, v-1),
$$

and so from (7) we deduce that

$$
n^{v} a_{n 0} \rightarrow(-1)^{v} B(r-2, v-1) / H(\alpha)^{v} B(r-1, v-1) .
$$

For $k>0$ we have $a_{n k}=D_{n k} / \Delta_{n}(r-k-1, \nu-k-1)$, where $D_{n k}$ is the determinant of the matrix obtained from $A_{n}(r-k-1, \nu-k-1)$ by replacing the first column by

$$
\begin{aligned}
\left(-c_{n, r-v-1}-\sum_{i=0}^{k-1} c_{n, r-i-1} a_{n i},-c_{n, r-v-2}\right. & -\sum_{i=0}^{k-1} c_{n, r-i-2} a_{n i}, \ldots, \\
& \left.-c_{n, r-2 v+k}-\sum_{i=0}^{k-1} c_{n, r-i-v+k} a_{n i}\right)^{T} .
\end{aligned}
$$

If we assume that $\lim _{n \rightarrow \infty} n^{v-i} a_{n i}$ exists for $i=0, \ldots, k-1$, then it is easy to see from (6) that the sequence $n^{(v+1-r)(v-k)} D_{n k}$ converges, and hence from (7) so does $n^{v-k} a_{n k}$, which completes the induction.

Setting $\lim _{n \rightarrow \infty} n^{v-k} a_{n k} \equiv L_{k} H(\alpha)^{k-v}$, we see from (4) that the constants $L_{k}$ must satisfy the linear system of equations

$$
\sum_{k=0}^{\nu-1} b_{r-k-j} L_{k}=-b_{r-v-j}, \quad 1 \leqq j \leqq \nu,
$$

which has a unique solution, namely

$$
L_{k}=(r-k-1) !(-1)^{\nu-k}\left(\begin{array}{l}
\nu \\
k
\end{array}\right) /(r-\nu-1) !
$$

and the lemma is proved. 
The importance of the constants $c_{n k}$ follows from the fact [5, proof of Theorem 1] that if $s_{n}(z)$ is the unique polynomial of degree $n$ which interpolates to the function $(z-\alpha)^{-\mu}, \mu>0$, in the $n+1$ roots of $\omega_{n}(z)$, then

$$
(z-\alpha)^{-\mu}-s_{n}(z)=\frac{\omega_{n}(z)}{\omega_{n}(\alpha)} \sum_{j=1}^{\mu} c_{n, \mu-j}(z-\alpha)^{-j} .
$$

The importance of the equations (4) will be evident from the proof of

THEOREM 2. Suppose $F(z)$ is a meromorphic function of the form

$$
F(z)=g(z)+\sum_{k=1}^{r} B_{k}(z-\alpha)^{-k},
$$

where the point $\alpha$ lies on $\Gamma_{\rho}(\rho>1), H(\alpha) \neq 0$, and $g(z)$ is analytic on the closed region $E_{o}+\Gamma_{\rho}$. For fixed $\nu, 0 \leqq \nu<r$, let the polynomials $Q_{n v}(z)$ be as in (3) with coefficients $a_{n k}$ defined in Lemma 1. If $P_{n v}(z)$ is the polynomial of degree $n$ which interpolates to the function $Q_{n v}(z) F(z)$ in the $n+1$ roots of $\omega_{n}(z)$ and $R_{n v}(z) \equiv P_{n v}(z) / Q_{n v}(z)$, then:

$$
\begin{aligned}
\left|F(z)-R_{n v}(z)\right| & \leqq A n^{r-2 v-1} / \rho^{n}, \text { for } z \text { on } E . \\
\operatorname{Lim}_{n \rightarrow \infty} \frac{R_{n v}(z) \omega_{n}(\alpha)}{n^{r-2 v-1} \omega_{n}(z)} & =\frac{\nu !(-1)^{v+1} H(\alpha)^{r-2 v-1}}{(r-\nu-1) !} \sum_{k=0}^{\nu} B_{r-k}(z-\alpha)^{k-2 v-1},
\end{aligned}
$$

uniformly for $z$ on each closed set exterior to $\Gamma_{\rho}$.

$$
\operatorname{Lim}_{n \rightarrow \infty} n^{-r} R_{n v}(\alpha)=\frac{\nu !(-1)^{v} H(\alpha)^{r} B_{r}}{(r-1) ! r(r-1) \cdots(r-v)} .
$$

Here and below constants $A$ are independent of $n$ and $z$ and may change from one inequality to another.

Proof. For $\nu=0$, Theorem 2 is a special case of [5, Theorems 2 and 3] so we need only consider $\nu>0$. Note that by (5) the sequence $Q_{n v}(z)$ converges uniformly on each compact subset of the plane to the function $(z-\alpha)^{\nu}$.

Write $P_{n v}(z)=\sum_{i=0}^{r} p_{n i}(z)$, where $p_{n 0}(z), p_{n 1}(z), \ldots, p_{n r}(z)$ are the polynomials of degree $n$ which interpolate respectively to the functions

$$
Q_{n v}(z) g(z), Q_{n v}(z) B_{1}(z-\alpha)^{-1}, \ldots, Q_{n v}(z) B_{r}(z-\alpha)^{-r}
$$

in the $n+1$ roots of $\omega_{n}(z)$. For the polynomials $p_{n 0}(z)$ we have by the Hermite formula

$$
Q_{n v}(z) g(z)-p_{n 0}(z)=\frac{1}{2 \pi i} \int_{\Gamma_{\tau}} \frac{\omega_{n}(z) Q_{n v}(t) g(t)}{\omega_{n}(t)(t-z)} d t, \quad z \text { in } E_{\tau}
$$

where $\tau(>\rho)$ is chosen so that $g(z)$ is analytic on the closed region $E_{\tau}+\Gamma_{\tau}$. Since the sequence $Q_{n v}(t) g(t)$ is uniformly bounded on $\Gamma_{\tau}$ and since the inequalities (2) imply

$$
\begin{array}{ll}
\left|\omega_{n}(z) / \omega_{n}(t)\right| \leqq A / \tau^{n}, & z \text { on } E, t \text { on } \Gamma_{\tau}, \\
\left|\omega_{n}(z) / \omega_{n}(t)\right| \leqq A \sigma^{n} / \tau^{n}, & z \text { on } \Gamma_{\sigma}, t \text { on } \Gamma_{\imath},
\end{array}
$$


it is easy to see, as in the proof of [5, Theorem 3], that

$$
\begin{gathered}
\left|Q_{n v}(z) g(z)-p_{n 0}(z)\right| \leqq A n^{r-2 v-1} / \rho^{n}, \quad z \text { on } E, \\
\lim _{n \rightarrow \infty} p_{n 0}(z) \omega_{n}(\alpha) / n^{r-2 v-1} \omega_{n}(z)=0,
\end{gathered}
$$

uniformly for $z$ on each closed set exterior to $\Gamma_{\rho}$.

Now consider the polynomials $p_{n i}(z), i>0$. From (8) we deduce that for $n \geqq \nu-i$

$$
Q_{n v}(z) B_{i}(z-\alpha)^{-i}-p_{n i}(z)=B_{i} \frac{\omega_{n}(z)}{\omega_{n}(\alpha)} \sum_{j=r+1-i}^{r} \lambda_{n j}(z-\alpha)^{r-i-j},
$$

where $\lambda_{n j} \equiv c_{n, r-v-j}+\sum_{k=0}^{\nu-1} a_{n k} c_{n, r-k-j}$. By our choice of the constants $a_{n k}$ we have $\lambda_{n j}=0$ for $1 \leqq j \leqq \nu$. Also, from (5) and (6) it follows that $n^{1+2 v-r} \lambda_{n j} \rightarrow 0$ for $j>v+1$ and that

$$
\lim _{n \rightarrow \infty} n^{1+2 v-r} \lambda_{n, v+1}=\frac{H(\alpha)^{r-2 v-1}}{(r-\nu-1) !} \sum_{k=0}^{m}(r-k-1) !(-1)^{v-k}\left(\begin{array}{l}
\nu \\
k
\end{array}\right) /(r-k-\nu-1) !,
$$

where $m \equiv \min (\nu, r-\nu-1)$. Thus $\left|\lambda_{n j}\right| \leqq A_{1} n^{r-2 v-1}, 1 \leqq j \leqq r$, and so from (12) we conclude that

$$
\left|Q_{n v}(z) B_{i}(z-\alpha)^{-i}-p_{n i}(z)\right| \leqq A_{2} n^{r-2 v-1 / \rho^{n}}, \quad z \text { on } E .
$$

The last inequality together with (10) yields

$$
\left|Q_{n v}(z) F(z)-P_{n v}(z)\right| \leqq A_{3} n^{r-2 v-1} / \rho^{n}, \quad z \text { on } E,
$$

which implies conclusion (i).

To prove (ii) note that

$$
Q_{n v}(z) B_{i}(z-\alpha)^{-i} \omega_{n}(\alpha) / n^{r-2 v-1} \omega_{n}(z) \rightarrow 0,
$$

uniformly for $z$ on each closed set exterior to $\Gamma_{\rho}$. Thus from (12) we deduce that

$$
\begin{aligned}
\lim _{n \rightarrow \infty} p_{n i}(z) \omega_{n}(\alpha) / n^{r-2 \nu-1} \omega_{n}(z) & =-l_{\nu} B_{i}(z-\alpha)^{r-i-\nu-1}, & & \text { for } i \geqq r-\nu, \\
& =0, & & \text { for } i<r-\nu,
\end{aligned}
$$

uniformly for $z$ on each closed set exterior to $\Gamma_{\rho}$, where $l_{v}$ is the right-hand member of (13). Using the fact that for any positive integer $q$

$$
\begin{aligned}
\sum_{k=0}^{q} k^{s}(-1)^{q-k}\left(\begin{array}{l}
q \\
k
\end{array}\right) & =q !, \quad \text { for } s=q, \\
& =0, \quad \text { for } s=0,1, \ldots, q-1,
\end{aligned}
$$

it is easy to show that $l_{v}=\nu !(-1)^{v} H(\alpha)^{r-2 v-1} /(r-\nu-1)$ !, and so from (11) and (15) we obtain

$$
\lim _{n \rightarrow \infty} \frac{P_{n v}(z) \omega_{n}(\alpha)}{n^{r-2 v-1} \omega_{n}(z)}=\frac{\nu !(-1)^{v+1} H(\alpha)^{r-2 v-1}}{(r-\nu-1) !} \sum_{i=r-v}^{r} B_{i}(z-\alpha)^{r-i-v-1},
$$

uniformly for $z$ on each closed set exterior to $\Gamma_{\rho}$, which implies (ii). 
Finally we consider the sequences $p_{n i}(\alpha)$. For $i=0$, it is immediate from (9) that $p_{n 0}(\alpha) \rightarrow 0$. For $i>0$, it has been shown [5, proof of Theorem 1] that $p_{n i}(\alpha)=B_{i} \lambda_{n, r-i}$. Hence from (5) and (6) we deduce that $n^{\nu-r} p_{n i}(\alpha) \rightarrow 0$ for $i<r$, and that

$$
\lim _{n \rightarrow \infty} n^{\nu-r} p_{n r}(\alpha)=\frac{H(\alpha)^{r-v} B_{r}}{(r-\nu-1) !} \sum_{k=0}^{\nu}(-1)^{\nu-k}\left(\begin{array}{l}
\nu \\
k
\end{array}\right) /(r-k) .
$$

It is easy to see from a partial fractions expansion that

$$
\sum_{k=0}^{v}(-1)^{\nu-k}\left(\begin{array}{l}
\nu \\
k
\end{array}\right) /(r-k)=\nu ! / r(r-1) \cdots(r-v)
$$

and so $n^{\nu-r} P_{n v}(\alpha) \rightarrow \nu ! H(\alpha)^{r-v} B_{r} /(r-\nu-1) ! r(r-1) \cdots(r-\nu)$. Since $Q_{n v}(\alpha)=a_{n 0}$, we can use (5) to obtain (iii)

$$
n^{-r} R_{n v}(\alpha)=n^{\nu-r} P_{n v}(\alpha) / n^{\nu} a_{n 0} \rightarrow \nu !(-1)^{\nu} H(\alpha)^{r} B_{r} /(r-1) ! r(r-1) \cdots(r-\nu),
$$

which completes the proof of the theorem.

Note that the inequalities (2) imply that $\left|\omega_{n}(\alpha) / \omega_{n}(z)\right| \leqq M_{0}$ for $z$ on $\Gamma_{\rho}$. Hence if $r-2 v-1>0$, then the limit (14) also holds for each $z(\neq \alpha)$ on $\Gamma_{\rho}$. By slightly modifying the proof of conclusion (ii) we therefore obtain

COROLlaRY 1. If $\nu<(r-1) / 2$, then the limit in conclusion (ii) of Theorem 2 holds uniformly for $z$ on each closed subset of $\Gamma_{\rho}$ which does not contain $\alpha$.

If, however, we have $r-2 \nu-1<0$, then $\lambda_{n j} \rightarrow 0$ for $1 \leqq j \leqq r$ and so the left-hand member of (12) converges to zero for each $z(\neq \alpha)$ on $\Gamma_{\rho}$. Thus we deduce

COROLlaRY 2. If $\nu>(r-1) / 2$, then the $R_{n v}(z)$ converge to $F(z)$ on $\Gamma_{\rho}-\{\alpha\}$, and for each closed subset $S$ of $\Gamma_{\rho}$ which does not contain $\alpha$ we have

$$
\left|F(z)-R_{n v}(z)\right| \leqq A n^{r-2 v-1}, \quad z \text { on } S .
$$

3. Approximating rational functions. We now use Theorem 2 to study the convergence of certain sequences of approximating rational functions. An easy consequence of conclusion (i) is

THEOREM 3. Let $f(z)$ be analytic on $E$, meromorphic with precisely $\mu(\geqq 0)$ poles in $E_{\rho}(1<\rho<\infty)$, and analytic on $\Gamma_{\rho}$ except for a pole of order $r$ in the point $\alpha \in \Gamma_{\rho}$, $\alpha$ not a critical point of the Green's function $G(z)$. Then for $0 \leqq \nu<r$ we have

$$
E_{n, \mu+v}(f) \leqq A n^{r-2 v-1} / \rho^{n}, \quad n>0 .
$$

Proof. Let $\pi(z)=z^{\mu}+a_{\mu-1} z^{\mu-1}+\cdots+a_{0}$ be the polynomial of the form indicated having as its zeros the $\mu$ poles of $f(z)$ in $E_{o}$. If $R_{n v}(z)$ is the rational function of type $(n, \nu)$ which is defined as in Theorem 2 for the function $F(z) \equiv \pi(z) f(z)$, then we have for $z$ on $E$

$$
\left|f(z)-R_{n v}(z) / \pi(z)\right| \leqq A_{1}\left|F(z)-R_{n v}(z)\right| \leqq A_{2} n^{r-2 v-1} / \rho^{n} .
$$

Since $R_{n v}(z) / \pi(z)$ is a rational function of type $(n, \mu+\nu)$, the inequality (16) follows.

We now state our main result. 


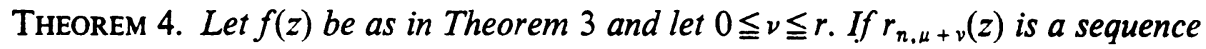
of rational functions of respective types $(n, \mu+\nu)$ which satisfy

$$
\left[\max \left|f(z)-r_{n, \mu+v}(z)\right| ; z \text { on } E\right]=o\left(n^{r-2 v+1} / \rho^{n}\right) \text { as } n \rightarrow \infty,
$$

then for $n$ sufficiently large each $r_{n, \mu+v}(z)$ has precisely $\mu+\nu$ finite poles, $\mu$ of which approach respectively the $\mu$ poles of $f(z)$ in $E_{o}$ and $\nu$ of which approach the point $\alpha$. Consequently the $r_{n, \mu+v}(z)$ converge uniformly to $f(z)$ on each closed subset of $E_{o}$ which contains no pole of $f(z)$.

In the proof of Theorem 4 and later theorems it is convenient to have for reference

LEMMA 2. Let $f(z)$ be as in Theorem 3 and suppose that $r_{n, \mu+v}(z)$ is a sequence of rational functions of respective types $(n, \mu+\nu)$ which satisfy for some real $\tau$

$$
\left[\max \left|f(z)-r_{n, \mu+v}(z)\right| ; z \text { on } E\right]=o\left(n^{\tau} / \rho^{n}\right) \text { as } n \rightarrow \infty .
$$

Let $q_{n}(z)$ be the monic polynomial whose zeros are the finite poles of $r_{n, u+v}(z)$, multiplicity included, and set $p_{n}(z) \equiv q_{n}(z) r_{n, \mu+v}(z)$. If the finite poles of the $r_{n, u+v}(z)$ are uniformly bounded, then $\lim _{n \rightarrow \infty} p_{n}(z) \omega_{n}(\alpha) / n^{\tau} \omega_{n}(z)=0$ uniformly for $z$ on each closed set exterior to $\Gamma_{\rho}$.

The $r_{n, u+v}(z)$ need not be defined for every $n$.

Proof. First note that the sequence $q_{n}(z)$ is uniformly bounded on each compact subset of the plane. This follows from the fact that the monic polynomials $q_{n}(z)$ have at most $\mu+\nu$ zeros and these zeros are uniformly bounded.

Now let $h(z) \equiv(z-\alpha)^{r} \pi(z) f(z)$, where $\pi(z)$ is defined as in the proof of Theorem 3 . Since $h(z)$ is analytic on $E_{\rho}+\Gamma_{\rho}$, the polynomials $h_{n}(z)$ of respective degrees $n$ of best uniform approximation to $h(z)$ on $E$ satisfy

$$
\limsup _{n \rightarrow \infty}\left[\max \left|h(z)-h_{n}(z)\right| ; z \text { on } E\right]^{1 / n}<1 / \rho .
$$

The last inequality and (18) imply that

$$
\left|(z-\alpha)^{r} \pi(z) r_{n, \mu+v}(z)-h_{n}(z)\right| \leqq \varepsilon_{n} n^{\tau} / \rho^{n}, \quad z \text { on } E,
$$

where $\varepsilon_{n} \rightarrow 0$, and hence

$$
\left|(z-\alpha)^{r} \pi(z) p_{n}(z)-q_{n}(z) h_{n}(z)\right| \leqq A \varepsilon_{n} n^{z} / \rho^{n}, \quad z \text { on } E .
$$

Since the function whose absolute value appears in the last inequality is a polynomial of degree $n+r+\mu+\nu$, we have by the Generalized Bernstein Lemma [1, p. 77] that

$$
\left|(z-\alpha)^{r} \pi(z) p_{n}(z)-q_{n}(z) h_{n}(z)\right| \leqq A_{1} \varepsilon_{n} n^{z} \sigma^{n} / \rho^{n}, \quad z \text { on } \Gamma_{\sigma} .
$$

If we choose $\sigma(>\rho)$ sufficiently close to $\rho$, then the $h_{n}(z)$ will be uniformly bounded on $\Gamma_{\sigma}[1$, p. 90] and hence

$$
\left|p_{n}(z)\right| \leqq A_{2} \varepsilon_{n} n^{\tau} \sigma^{n} / \rho^{n}+A_{3}, \quad z \text { on } \Gamma_{\sigma} .
$$


Since $\left|\omega_{n}(\alpha) / \omega_{n}(z)\right| \leqq A \rho^{n} / \sigma^{n}$ for $z$ on $\Gamma_{\sigma}$, we conclude that $p_{n}(z) \omega_{n}(\alpha) / n^{\tau} \omega_{n}(z) \rightarrow 0$ uniformly for $z$ on or exterior to $\Gamma_{\sigma}$ and so the lemma follows from the arbitrariness of $\sigma$.

Proof of Theorem 4. For $\nu=0$ the theorem reduces to a special case of Theorem 1, so suppose that $\nu>0$ and write $r_{n, \mu+v}(z)=p_{n}(z) / q_{n}(z)$ as in Lemma 2 .

We assume at first that the finite poles of the $r_{n, \mu+v}(z)$ are uniformly bounded so that the sequence $q_{n}(z)$ forms a normal family in the whole plane. Let $q(z)$ be any limit function of this sequence and note that $q(z)$ must be a polynomial of the form $q(z)=z^{n}+c_{1} z^{n-1}+\cdots+c_{\eta}$, where $0 \leqq \eta \leqq \mu+\nu$. Walsh has shown, as a consequence of his proof of Theorem 1 , that the polynomial $\pi(z)$ must be a factor of $q(z)$. Thus if we show that $(z-\alpha)^{v}$ is also a factor, then it follows from the form of $q(z)$ that $q(z) \equiv(z-\alpha)^{v} \pi(z)$.

Taking $F(z)=\pi(z) f(z)$, we let $R_{n, v-1}(z)=P_{n, v-1}(z) / Q_{n, v-1}(z)$ be the rational functions of respective types $(n, \nu-1)$ which are defined in Theorem 2. From (17) and conclusion (i) of Theorem 2 there follows

$$
\left|R_{n, v-1}(z)-\pi(z) r_{n, \mu+v}(z)\right| \leqq A_{1} n^{r-2 v+1} / \rho^{n}, \quad z \text { on } E,
$$

and on multiplying this last inequality by $\left|q_{n}(z) Q_{n, v-1}(z)\right|$ and applying the Bernstein lemma we deduce that the sequence

$$
\phi_{n}(z) \equiv\left(q_{n}(z) P_{n, v-1}(z)-\pi(z) Q_{n, v-1}(z) p_{n}(z)\right) \omega_{n}(\alpha) / n^{r-2 v+1} \omega_{n}(z)
$$

is uniformly bounded on each $\Gamma_{\sigma}(\sigma>1)$ and hence on each compact subset of $K$. Now by Lemma 2 the sequence $\pi(z) Q_{n, v-1}(z) p_{n}(z) \omega_{n}(\alpha) / n^{r-2 v+1} \omega_{n}(z)$ converges to zero at each finite point exterior to $\Gamma_{\rho}$, and so from conclusion (ii) of Theorem 2 we see that some subsequence of the $\phi_{n}(z)$ converges to the function

$$
\phi(z) \equiv \frac{(\nu-1) !(-1)^{\nu} H(\alpha)^{r-2 v+1}}{(r-v) !} q(z) \sum_{k=0}^{\nu-1} B_{r-k}(z-\alpha)^{k-v}
$$

for $z$ exterior to $\Gamma_{\rho}$, where $\sum_{1}^{r} B_{k}(z-\alpha)^{-k}$ is the singular part of the pole $\alpha$ of $F(z)$. But the family $\phi_{n}(z)$ is normal in $K-\{\infty\}$ and hence $\phi(z)$ must be analytic in this domain and in particular at $z=\alpha$. Therefore since $B_{r} \neq 0,(z-\alpha)^{v}$ must be a factor of $q(z)$ and so $q(z) \equiv(z-\alpha)^{v} \pi(z)$.

Thus the only limit function of the $q_{n}(z)$ is $(z-\alpha)^{v} \pi(z)$ and hence $q_{n}(z) \rightarrow$ $(z-\alpha)^{v} \pi(z)$ uniformly for $z$ on each compact subset of the plane. The first part of Theorem 4 now follows from Hurwitz's theorem provided we show that the finite poles of the $r_{n, \mu+v}(z)$ are uniformly bounded.

Following Walsh's method we suppose to the contrary that the sequence $r_{n, u+v}(z)$, possesses a subsequence, which we continue to denote by $r_{n, \mu+v}(z)$, with the property that precisely $\lambda$ of the finite poles of the $r_{n, u+v}(z)$ approach infinity while the remaining $\mu+\nu-\lambda$ (or fewer) poles are uniformly in modulus less than some $R$. Let $\beta_{n 1}, \beta_{n 2}, \ldots, \beta_{n \lambda}$ be those finite poles of $r_{n, \mu+v}(z)$ which lie outside the circle of radius $R$, and set $\psi_{n}(z) \equiv \prod_{i=1}^{\lambda}\left(1-z \beta_{n i}^{-1}\right), r_{n, \mu+v}^{*}(z) \equiv \psi_{n}(z) r_{n, \mu+v}(z)$. On 
multiplying the inequalities (19) and (20) by $\left|\psi_{n}(z)\right|$ and applying to the $r_{n, u+v}^{*}(z)$ the same reasoning which was used for the $r_{n, \mu+v}(z)$ we see that for $n$ sufficiently large each $r_{n, \mu+v}^{*}(z)$ has at least $\mu+\nu$ finite poles. But this is impossible since the $r_{n, u+v}^{*}(z)$ have at most $\mu+\nu-\lambda$ finite poles and the first part of the theorem is proved.

As another consequence of his proof of Theorem 1, Walsh mentions [11, Corollary 4] that any sequence of rational functions $r_{n, \mu+v}(z)$ of respective types $(n, \mu+\nu)$ which satisfy

$$
\limsup _{n \rightarrow \infty}\left[\max \left|f(z)-r_{n, \mu+v}(z)\right| ; z \text { on } E\right]^{1 / n} \leqq 1 / \rho
$$

must converge uniformly to $f(z)$ on each compact subset of $E_{\rho}$ which contains no limit points of poles of the $r_{n, \mu+v}(z)$. Since the rational functions of Theorem 4 have no limit points of poles other than the poles of $f(z)$, the second part of the theorem follows and the proof is complete.

Concerning the divergence at $\alpha$ of the approximating sequence $r_{n, u+v}(z)$ we prove

THEOREM 5. Let $f(z)$ be as in Theorem 3 and suppose the rational functions $r_{n, u+v}(z)$ of respective types $(n, \mu+\nu), 0 \leqq \nu<r$, satisfy

$$
\left[\max \left|f(z)-r_{n, \mu+v}(z)\right| ; z \text { on } E\right]=o\left(n^{r-2 v} / \rho^{n}\right) \text { as } n \rightarrow \infty .
$$

Then we have

$$
\lim _{n \rightarrow \infty} n^{-r} r_{n, \mu+v}(\alpha)=\frac{v !(-1)^{v} H(\alpha)^{r} B}{(r-1) ! r(r-1) \cdots(r-v)}
$$

where $B \equiv \lim _{z \rightarrow \alpha}(z-\alpha)^{r} f(z)$.

Proof. Using the same notation as in the previous proof we first show that the sequence $n^{v} q_{n}(\alpha)$ converges to a nonzero limit. This is obvious if $\nu=0$, so assume $\nu>0$.

Let $R_{n, v-1}^{*}(z)=P_{n, v-1}^{*}(z) / Q_{n, v-1}^{*}(z)$ be the rational functions of respective types $(n, v-1)$ which are defined as in Theorem 2 for the function $F^{*}(z) \equiv(z-\alpha) \pi(z) f(z)$. Since $F^{*}(z)$ has a pole of order $r-1$ at $\alpha$, Theorem 2 implies that

and hence

$$
\left|F^{*}(z)-R_{n, v-1}^{*}(z)\right| \leqq A n^{r-2 v} / \rho^{n}, \quad z \text { on } E,
$$

$$
\left|R_{n, v-1}^{*}(z)-(z-\alpha) \pi(z) r_{n, \mu+v}(z)\right| \leqq A_{1} n^{r-2 v} / \rho^{n}, \quad z \text { on } E .
$$

Reasoning as in the proof of Theorem 4 we deduce from (23) that the sequence

$$
\phi_{n}^{*}(z) \equiv\left(q_{n}(z) P_{n, v-1}^{*}(z)-(z-\alpha) \pi(z) Q_{n, v-1}^{*}(z) p_{n}(z)\right) \omega_{n}(\alpha) / n^{r-2 v} \omega_{n}(z)
$$

forms a normal family in $K-\{\infty\}$. Moreover since $q_{n}(z) \rightarrow(z-\alpha)^{v} \pi(z)$, we see from Lemma 2 and conclusion (ii) of Theorem 2 that for $z$ exterior to $\Gamma_{0}$ and consequently for $z$ in $K-\{\infty\}$

$$
\lim _{n \rightarrow \infty} \phi_{n}^{*}(z)=L \pi(z) \sum_{k=0}^{\nu-1} B_{r-k-1}^{*}(z-\alpha)^{k},
$$


where $L \equiv(\nu-1) !(-1)^{\nu} H(\alpha)^{r-2 v} /(r-\nu-1) !$ and $\sum_{1}^{r-1} B_{k}^{*}(z-\alpha)^{-k}$ is the singular part of the pole $\alpha$ of $F^{*}(z)$. Thus

$$
\phi_{n}^{*}(\alpha)=n^{2 v-r} q_{n}(\alpha) P_{n, v-1}^{*}(\alpha) \rightarrow L \pi(\alpha) B_{r-1}^{*} \neq 0,
$$

and since the sequence $n^{\nu-r} P_{n, v-1}^{*}(\alpha)$ converges to a nonzero limit, the same must be true of the sequence $n^{v} q_{n}(\alpha)$.

Now consider the rational functions $R_{n v}(z)=P_{n v}(z) / Q_{n v}(z)$ of respective types $(n, \nu)$ which are defined as in Theorem 2 for the function $\pi(z) f(z)$. Since

$$
\left|q_{n}(z) P_{n v}(z)-\pi(z) Q_{n v}(z) p_{n}(z)\right| \leqq \varepsilon_{n} n^{r-2 v} / \rho^{n}, \quad z \text { on } E,
$$

where $\varepsilon_{n} \rightarrow 0$, the Bernstein lemma implies that

$$
\left|q_{n}(\alpha) P_{n v}(\alpha)-\pi(\alpha) Q_{n v}(\alpha) p_{n}(\alpha)\right| \leqq A \varepsilon_{n} n^{r-2 v} .
$$

We have shown that for $n$ sufficiently large the sequence

$$
n^{2 v}\left|Q_{n v}(\alpha) q_{n}(\alpha)\right|=n^{v}\left|a_{n 0}\right| n^{\nu}\left|q_{n}(\alpha)\right|
$$

is uniformly bounded away from zero, and so (24) yields

$$
\left|R_{n v}(\alpha)-\pi(\alpha) r_{n, \mu+v}(\alpha)\right| \leqq A_{1} \varepsilon_{n} n^{r} .
$$

Hence $n^{-r}\left(R_{n v}(\alpha)-\pi(\alpha) r_{n, \mu+v}(\alpha)\right) \rightarrow 0$ and (22) follows from conclusion (iii) of Theorem 2.

We remark that Theorem 5 is best possible in the sense that (22) may not hold if in (21) we replace $o\left(n^{r-2 v} / \rho^{n}\right)$ by $O\left(n^{r-2 v} / \rho^{n}\right)$. Indeed, the sequence $R_{n, v-1}^{*}(z) /(z-\alpha) \pi(z)$ is of the latter degree of approximation to $f(z)$ on $E$ but clearly does not satisfy (22).

Concerning divergence at points exterior to $\Gamma_{\rho}$ we have

THEOREM 6. Let $f(z)$ be as in Theorem 3 and suppose the rational functions $r_{n, \mu+v}(z)$ of respective types $(n, \mu+\nu), 0 \leqq \nu<r$, satisfy

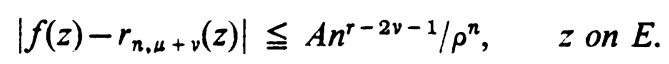

Then except for a finite number of points the inequality

$$
\underset{n \rightarrow \infty}{\lim \sup }\left|r_{n, u+v}(z)\right| \rho^{n} / n^{r-2 v-1} \exp (n G(z))>0
$$

holds for each $z$ exterior to $\Gamma_{\rho}$. Consequently the $r_{n, \mu+v}(z)$ can converge in at most a finite number of points exterior to $\Gamma_{\rho}$.

The $r_{n, u+v}(z)$ need not be defined for every $n$.

Proof. Taking $R_{n v}(z)$ as in the previous proof it follows from (25) that the sequence

$$
\left(q_{n}(z) P_{n v}(z)-\pi(z) Q_{n v}(z) p_{n}(z)\right) \omega_{n}(\alpha) / n^{r-2 v-1} \omega_{n}(z)
$$


is uniformly bounded on each compact subset of $K$. Thus from conclusion (ii) of Theorem 2 we deduce that the sequence

$$
T_{n}(z) \equiv \pi(z) Q_{n v}(z) p_{n}(z) \omega_{n}(\alpha) / n^{r-2 v-1} \omega_{n}(z)
$$

forms a normal family in the exterior of $\Gamma_{\rho}$. Since $q_{n}(z) \rightarrow(z-\alpha)^{v} \pi(z)$, each limit function of the $T_{n}(z)$ must be of the form

$$
\psi(z)+\frac{\nu !(-1)^{\nu+1} H(\alpha)^{r-2 \nu-1}}{(r-\nu-1) !} \pi(z) \sum_{k=0}^{v} B_{r-k}(z-\alpha)^{k-1},
$$

where $\psi(z)$ is analytic in $K$ except possibly for a pole at infinity, and $\sum_{1}^{r} B_{k}(z-\alpha)^{-k}$ is the singular part of the pole $\alpha$ of $\pi(z) f(z)$. It is easy to see from (27) that any limit function $T(z)$ of the $T_{n}(z)$ can have at most a finite number of zeros on or exterior to $\Gamma_{\rho}$. But if $z$ lies exterior to $\Gamma_{\rho}$ and is not a zero of $T(z)$ we have

$$
0<\limsup _{n \rightarrow \infty}\left|T_{n}(z)\right| \leqq \limsup _{n \rightarrow \infty} \frac{A\left|r_{n, \mu+v}(z)\right| \rho^{n}}{n^{r-2 v-1} \exp (n G(z))},
$$

which proves the theorem.

Corollaries 1 and 2 yield

COROLLARY 3. If under the hypotheses of Theorem 6 we have $\nu<(r-1) / 2$, then except for a finite number of points the inequality (26) holds for each $z$ on $\Gamma_{0}$ and hence the $r_{n, \mu+v}(z)$ can converge in at most a finite number of points on $\Gamma_{\rho}$.

If $\nu>(r-1) / 2$, then the $r_{n, \mu+v}(z)$ converge to $f(z)$ on $\Gamma_{\rho}-\{\alpha\}$, and for each closed subset $S$ of $\Gamma_{\rho}$ which does not contain $\alpha$ we have

$$
\left|f(z)-r_{n, u+v}(z)\right| \leqq A n^{r-2 v-1}, \quad z \text { on } S .
$$

Proof. If $\nu<(r-1) / 2$, Corollary 1 implies that the sequence $T_{n}(z)$ possesses a subsequence which converges to a function of the form (27) for $z$ on $\Gamma_{\rho}-\{\alpha\}$. Thus by applying the reasoning of the previous proof we deduce the first part of the corollary.

Now suppose $\nu>(r-1) / 2$ and note that for $n$ sufficiently large and $z$ on $S$ we have $\left|q_{n}(z) Q_{n v}(z)\right| \geqq m>0$. The inequality

$$
\left|q_{n}(z) P_{n v}(z)-\pi(z) Q_{n v}(z) p_{n}(z)\right| \leqq A n^{r-2 v-1}, \quad z \text { on } \Gamma_{\rho},
$$

therefore implies

$$
\left|R_{n v}(z)-\pi(z) r_{n, \mu+v}(z)\right| \leqq A_{1} n^{r-2 v-1}, \quad z \text { on } S,
$$

and so (28) follows from Corollary 2 which completes the proof.

We now show that the degree of approximation indicated in Theorem 3 is best possible.

THEOREM 7. If $f(z)$ is defined as in Theorem 3 and $0 \leqq \nu<r$, then there exists a constant $A_{1}$ such that

$$
n^{1+2 v-r} \rho^{n} E_{n, \mu+v}(f) \geqq A_{1}>0, \quad n>0 .
$$


Proof. The contrary assumption would imply that there exists an increasing sequence of positive integers $k$ for which

$$
\left[\max \left|f(z)-W_{k, \mu+v}(z)\right| ; z \text { on } E\right]=o\left(k^{r-2 v-1} / \rho^{k}\right) \text { as } k \rightarrow \infty,
$$

where $W_{k, \mu+v}(z)$ is a rational function of type $(k, \mu+v)$ of best uniform approximation to $f(z)$ on $E$. But $W_{k, \mu+\nu}(z)$ is also of type $(k, \mu+\nu+1)$ and so Theorem 4 implies that for $k$ sufficiently large $W_{k, \mu+\nu}(z)$ has $\mu+\nu+1$ finite poles which is impossible.

We remark that if the point $\alpha$ is a critical point of $G(z)$, then for $\nu=0$ the degree of approximation indicated in (16) can be improved [5, Theorem 7] so that (29) need not hold.

4. Several boundary poles. Although the methods of $\$ 3$ do not lead to a general result on the convergence of the rows of the Walsh array for a function $f(z)$ with poles in several points on $\Gamma_{\rho}$, the methods are useful in some special cases which we now consider. As a generalization of Theorem 4 we have

THEOREM 8. Let $f(z)$ be analytic on $E$, meromorphic with precisely $\mu(\geqq 0)$ poles in $E_{\rho}(1<\rho<\infty)$, and analytic on $\Gamma_{\rho}$ except for poles in the distinct points $\alpha_{1}, \alpha_{2}, \ldots, \alpha_{t}$ on $\Gamma_{\rho}$. Suppose that each of the poles of $f(z)$ on $\Gamma_{\rho}$ is of the same order $r$ and that no $\alpha_{i}$ is a critical point of $G(z)$. If $r_{n, u+t v}(z)$ is a sequence of rational functions of respective types $(n, \mu+t \nu), 0 \leqq \nu \leqq r$, which satisfy

$$
\left[\max \left|f(z)-r_{n, u+t v}(z)\right| ; z \text { on } E\right]=o\left(n^{r-2 v+1} / \rho^{n}\right) \quad \text { as } n \rightarrow \infty,
$$

then for $n$ sufficiently large each $r_{n, \mu+t v}(z)$ has precisely $\mu+t v$ finite poles, $\mu$ of which approach respecticeiy the $\mu$ poles of $f(z)$ in $E_{0}$ and $\nu$ of which approach each of the points $\alpha_{i}$.

Consequently the $r_{n, u+t v}(z)$ converge uniformly to $f(z)$ on each closed subset of $E_{\rho}$ which contains no pole of $f(z)$.

Proof. Let $\pi(z)$ be the monic polynomial of degree $\mu$ whose zeros are the poles of $f(z)$ in $E_{\rho}$, and write

$$
\pi(z) f(z)=g(z)+\sum_{i=1}^{t} S_{i}(z)
$$

where $g(z)$ is analytic on $E_{\rho}+\Gamma_{\rho}$ and $S_{i}(z)=\sum_{k=1}^{r} B_{k, i}\left(z-\alpha_{i}\right)^{-k}$ is the singular part of the pole $\alpha_{i}$ of $\pi(z) f(z)$. Taking $\alpha=\alpha_{i}$ and

$$
\begin{aligned}
F(z) & =g(z)+S_{1}(z), & & \text { for } i=1, \\
& =S_{i}(z), & & \text { for } i>1,
\end{aligned}
$$

let $R_{n, v-1}^{(i)}(z)=P_{n, v-1}^{\{i\}}(z) / Q_{n, v-1}^{(i)}(z)$ be the rational functions of respective types $(n, v-1)$ which are defined by Theorem 2 . 
Set

$$
R_{n}(z) \equiv \sum_{i=1}^{t} R_{n, v-1}^{(i)}(z), \quad Q_{n}(z) \equiv \prod_{i=1}^{t} Q_{n, v-1}^{(i)}(z)
$$

and let $P_{n}(z) \equiv Q_{n}(z) R_{n}(z)$. Note that the $R_{n}(z)$ are rational functions of respective types $(n+(t-1)(v-1), t(v-1))$ which by Theorem 2 satisfy

$$
\left|\pi(z) f(z)-R_{n}(z)\right| \leqq A n^{r-2 v+1} / \rho^{n}, \quad z \text { on } E .
$$

Furthermore, since the inequalities (2) imply that

$$
0<M_{1} \leqq\left|\omega_{n}\left(\alpha_{1}\right) / \omega_{n}\left(\alpha_{i}\right)\right| \leqq M_{2}<\infty, \quad n>0,
$$

it follows from conclusion (ii) of Theorem 2 that each subsequence of

$$
\left\{R_{n}(z) \omega_{n}\left(\alpha_{1}\right) / n^{r-2 v+1} \omega_{n}(z)\right\}
$$

possesses a subsequence which converges uniformly on each compact set exterior to $\Gamma_{\rho}$ to a function of the form

$$
\frac{(\nu-1) !(-1)^{v}}{(r-v) !} \sum_{i=1}^{t} \lambda_{i} H\left(\alpha_{i}\right)^{r-2 v+1} \sum_{k=0}^{\nu-1} B_{r-k, i}\left(z-\alpha_{i}\right)^{k-2 v+1},
$$

where the constants $\lambda_{i}$ are nonzero.

We can now proceed as in the proof of Theorem 4 by writing $r_{n, \mu+t v}(z)=p_{n}(z) / q_{n}(z)$ and showing that if $q(z)$ is a limit function of the $q_{n}(z)$, then $q(z) \equiv \pi(z) \prod_{1}^{t}\left(z-\alpha_{i}\right)^{v}$. Indeed, from (31) we deduce that the sequence

$$
\left(q_{n}(z) P_{n}(z)-\pi(z) Q_{n}(z) p_{n}(z)\right) \omega_{n}\left(\alpha_{1}\right) / n^{\gamma-2 v+1} \omega_{n}(z)
$$

is uniformly bounded on each compact subset of $K$. Since Lemma 2 is obviously valid in this case, it then follows that $\Phi(z) q(z) \prod_{1}^{t}\left(z-\alpha_{i}\right)^{v-1}$ must be analytic at each $\alpha_{i}$, where $\Phi(z)$ is a function of the form (32). Thus $\prod_{1}^{t}\left(z-\alpha_{i}\right)^{v}$ must be a factor of $q(z)$ and so $q(z) \equiv \pi(z) \prod_{1}^{t}\left(z-\alpha_{i}\right)^{\nu}$. The remainder of the proof is identical with that of Theorem 4 and is therefore omitted.

Theorems 3, 5, 6, and 7 admit similar extensions, but we only state

THEOREM 9. Let $f(z)$ be as in Theorem 8 and let $0 \leqq \lambda<$ tr. Then there exist constants $A_{1}, A_{2}$ such that

$$
0<A_{1} \leqq n^{1+2 v-r} \rho^{n} E_{n, \mu+\lambda}(f) \leqq A_{2}<\infty, \quad n>0,
$$

where $[\nu \equiv \lambda / t]$ is the greatest integer less than or equal to $\lambda / t$.

We conclude with

THEOREM 10. Let $f(z)$ be analytic on $E$, meromorphic with precisely $\mu(\geqq 0)$ poles in $E_{\rho}$, and analytic on $\Gamma_{\rho}$ except for poles in the two distinct points $\alpha_{1}, \alpha_{2}$ on $\Gamma_{\rho}$ of respective orders $r_{1}, r_{2}$. If neither $\alpha_{1}$ nor $\alpha_{2}$ is a critical point of $G(z)$ and if one $r_{i}$ is an even integer and the other is an odd integer, then the row' $\mu+1$ through $\mu+r_{1}+r_{2}+1$ 
of the Walsh array for $f(z)$ on $E$ converge uniformly to $f(z)$ on each closed subset of $E_{\rho}$ which contains no pole of $f(z)$.

In particular suppose that $r_{1}=2 p$ and $r_{2}=2 q-1$, where $p \geqq q$, and let $\nu_{0} \equiv p-q+1$, $\nu_{1} \equiv p+3 q-1$. Then for $n$ sufficiently large each of the best approximating rational functions $W_{n, \mu+v}(z), 0 \leqq \nu \leqq r_{1}+r_{2}$, has precisely $\mu+\nu$ finite poles, $\mu$ of which approach respectively the $\mu$ poles of $f(z)$ in $E_{\rho}$ and $\nu$ of which approach the points $\alpha_{i}$ as follows: if $\nu<\nu_{0}$, then all of the $\nu$ poles approach the point $\alpha_{1}$; if $\nu_{0} \leqq \nu<\nu_{1}$, then $\tau \equiv \nu_{0}+$ $\left[\left(\nu-\nu_{0}\right) / 2\right]$ poles approach the point $\alpha_{1}$ and $\nu-\tau$ poles approach $\alpha_{2}$; if $\nu \geqq \nu_{1}$, then $\nu-r_{2}$ poles approach $\alpha_{1}$ and $r_{2}$ poles approach $\alpha_{2}$.

Proof. For convenience we assume $\mu=0$. Write

$$
f(z)=g(z)+S_{1}(z)+S_{2}(z),
$$

where $g(z)$ is analytic on $E_{\rho}+\Gamma_{\rho}$ and $S_{i}(z)=\sum_{k=1}^{r_{i}} B_{k, i}\left(z-\alpha_{i}\right)^{-k}$ is the singular part of the pole of $f(z)$ at $\alpha_{i}$. Let $R_{n j}^{(i)}(z), 0 \leqq j<r_{i}$, be the rational function of type $(n, j)$ which is defined by Theorem 2 for $\alpha=\alpha_{i}$ and $F(z)=g(z)+S_{1}(z)$ for $i=1, F(z)=S_{2}(z)$ for $i=2$.

First suppose $0<\nu<\nu_{0}$, so that $r_{1}-2 \nu-1>r_{2}-1$. From Theorems 2 and 3 we obtain the inequalities

$$
\begin{gathered}
E_{n v}(f) \leqq E_{n v}\left(g+S_{1}\right)+E_{n-v, 0}\left(S_{2}\right) \leqq A n^{r_{1}-2 v-1} / \rho^{n}, \\
\left|W_{n v}(z)-R_{n, v-1}^{(1)}(z)-R_{n 0}^{\{2 \gamma}(z)\right| \leqq A_{1} n^{r_{1}-2 v+1} / \rho^{n}, \quad z \text { on } E .
\end{gathered}
$$

Also from conclusion (ii) of Theorem 2 we deduce that

$$
\lim _{n \rightarrow \infty} \frac{\left(R_{n, v-1}^{(1)}(z)+R_{n 0}^{(2)}(z)\right) \omega_{n}\left(\alpha_{1}\right)}{n^{r_{1}-2 v+1} \omega_{n}(z)}=C \sum_{k=0}^{v-1} B_{r_{1}-k, 1}\left(z-\alpha_{1}\right)^{k-2 v+1},
$$

uniformly on each closed set exterior to $\Gamma_{\rho}$, where the constant $C$ is nonzero. As can readily be seen from the proof of Theorem 4 the conditions (34), (35), and (36) are sufficient to guarantee that for $n$ sufficiently large each $W_{n v}(z)$ has precisely $v$ finite poles, and these poles approach the point $\alpha_{1}$.

Now suppose $\nu_{0} \leqq \nu<\nu_{1}$ so that $\tau<r_{1}$ and $\sigma \equiv \nu-\tau \leqq r_{2}$. Let

and note that

$$
\lambda \equiv \max \left(r_{1}-2 \tau-1, r_{2}-2 \sigma-1\right)
$$

$$
\lambda<\min \left(r_{1}-2 \tau+1, r_{2}-2 \sigma+1\right)
$$

Clearly we have the inequalities

$$
\begin{gathered}
E_{n v}(f) \leqq E_{n-\sigma . \tau}\left(g+S_{1}\right)+E_{n-\tau . \sigma}\left(S_{2}\right) \leqq A n^{\lambda} / \rho^{n}, \\
\left|W_{n v}(z)-R_{n, \tau-1}^{\{1\}}(z)-R_{n \sigma}^{\{2\}}(z)\right| \leqq A_{1} n^{r_{1}-2 \tau+1} / \rho^{n}, \quad z \text { on } E, \\
\left|W_{n v}(z)-R_{n \tau}^{\{1\}}(z)-R_{n, \sigma-1}^{\{2\}}(z)\right| \leqq A_{2} n^{r_{2}-2 \sigma+1} / \rho^{n}, \quad z \text { on } E,
\end{gathered}
$$

where we take $R_{n, r_{i}}^{(i)}(z)$ to be a rational function of type $\left(n, r_{i}\right)$ of best uniform 
approximation on $E$ to the function $g(z)+S_{1}(z)$ for $i=1, S_{2}(z)$ for $i=2$. Therefore since

$$
\lim _{n \rightarrow \infty} \frac{\left(R_{n, \tau}^{\{1\}}-1(z)+R_{n \sigma}^{\{2\}}(z)\right) \omega_{n}\left(\alpha_{1}\right)}{n^{\tau_{1}-2 \tau+1} \omega_{n}(z)}=C_{1} \sum_{k=0}^{\tau-1} B_{r_{1}-k, 1}\left(z-\alpha_{1}\right)^{k-2 \imath+1}
$$

$C_{1} \neq 0$, for $z$ exterior to $\Gamma_{\rho}$, it follows that at least $\tau$ of the poles of the $W_{n v}(z)$ approach the point $\alpha_{1}$. Similarly since

$$
\lim _{n \rightarrow \infty} \frac{\left(R_{n \tau}^{(1)}(z)+R_{n, \sigma-1}^{(2)}(z)\right) \omega_{n}\left(\alpha_{2}\right)}{n^{\tau_{2}-2 \sigma+1} \omega_{n}(z)}=C_{2} \sum_{k=0}^{\sigma-1} B_{r_{2}-k, 2}\left(z-\alpha_{2}\right)^{k-2 \sigma+1}
$$

$C_{2} \neq 0$, for $z$ exterior to $\Gamma_{\rho}$, we deduce that at least $\sigma$ of the poles of the $W_{n v}(z)$ approach the point $\alpha_{2}$. But $\tau+\sigma=\nu$, and so the second part of the theorem follows for $\nu_{0} \leqq \nu<\nu_{1}$.

Finally suppose $\nu \geqq \nu_{1}$ so that $r_{1}-2\left(\nu-r_{2}\right)-1<1-r_{2}$. Then we have

$$
\begin{gathered}
E_{n v}(f) \leqq E_{n-r_{2}, v-r_{2}}\left(g+S_{1}\right)+E_{n+r_{2}-v, r_{2}}\left(S_{2}\right) \leqq A n^{r_{1}-2\left(v-r_{2}\right)-1 / \rho^{n},} \\
\left|W_{n v}(z)-R_{n, v-r_{2}-1}^{(1)}(z)-R_{n, r_{2}}^{\{2\}}(z)\right| \leqq A_{1} n^{r_{1}-2\left(v-r_{2}\right)+1} / \rho^{n}, \quad z \text { on } E, \\
\left|W_{n v}(z)-R_{n, r_{1}}^{\{1\}}(z)-R_{n, r_{2}-1}^{\{2\}}(z)\right| \leqq A_{2} n^{1-r_{2}} / \rho^{n}, \quad z \text { on } E .
\end{gathered}
$$

But for $z$ exterior to $\Gamma_{o}$ we also have

$$
\begin{gathered}
\lim _{n \rightarrow \infty} \frac{\left(R_{n, v-r_{2}-1}^{\{1\}}(z)+R_{n, r_{2}}^{\{2\}}(z)\right) \omega_{n}\left(\alpha_{1}\right)}{n^{r_{1}-2\left(v-r_{2}\right)+1} \omega_{n}(z)}=\xi_{1} \sum_{k=0}^{v-r_{2}-1} B_{r_{1}-k, 1}\left(z-\alpha_{1}\right)^{k-2\left(v-r_{2}\right)+1}, \\
\lim _{n \rightarrow \infty} \frac{\left(R_{n, r_{1}}^{\{1\}}(z)-R_{n, r_{2}-1}^{\{2\}}(z)\right) \omega_{n}\left(\alpha_{2}\right)}{n^{1-r_{2} \omega_{n}(z)}}=\xi_{2} \sum_{k=0}^{r_{2}-1} B_{r_{2}-k, 2}\left(z-\alpha_{2}\right)^{k-2 r_{2}+1}
\end{gathered}
$$

where the constants $\xi_{1}, \xi_{2}$ are nonzero. Thus we deduce that $\nu-r_{2}$ of the poles of the $W_{n v}(z)$ approach $\alpha_{1}$ while $r_{2}$ of the poles approach $\alpha_{2}$, which completes the proof of the second part of the theorem. Analogous reasoning can be used to establish the asymptotic behavior of the poles of the $W_{n v}(z)$ for the case where the larger of $r_{1}$ and $r_{2}$ is an odd integer and so the first part of the theorem follows.

\section{REFERENCES}

1. J. L. Walsh, Interpolation and approximation by rational functions in the complex domain, 3rd ed., Amer. Math. Soc. Colloq. Publ., Vol. 20, Amer. Math. Soc., Providence, R. I., 1960.

2. - On approximation to an analytic function by rational functions of best approximation, Math. Z. 38 (1934), 163-176.

3. - "The convergence of sequences of rational functions of best approximation with some free poles," Approximation of functions, H. L. Garabedian, Editor, Elsevier, Amsterdam, 1965.

4. R. de Montessus de Ballore, Sur les fractions continues algébriques, Bull. Soc. Math. France 30 (1902), 28-36.

5. E. B. Saff, Polynomials of interpolation and approximation to meromorphic functions, Trans. Amer. Math. Soc. 143 (1969), 509-522. 
6. R. Wilson, Divergent continued fractions and polar singularities, Proc. London Math. Soc. 26 (1927), 159-168.

7. - Divergent continued fractions and polar singularities. II, Proc. London Math. Soc. 27 (1928), 497-512.

8. - Divergent continued fractions and polar singularities. III, Proc. London Math. Soc. 28 (1928), 128-145.

9. J. L. Walsh, A sequence of rational functions with applications to approximation by bounded analytic functions, Duke Math. J. 30 (1963), 177-190.

10. O. Perron, Die Lehre von den Kettenbrüchen, 2nd ed., Teubner, Leipzig, 1929.

11. J. L. Walsh, Surplus free poles of approximating rational functions, Proc. Nat. Acad. Sci. U.S.A. 52 (1964), 896-901.

\section{IMPERIAL COLLEGE,}

LONDON, ENGLAND 